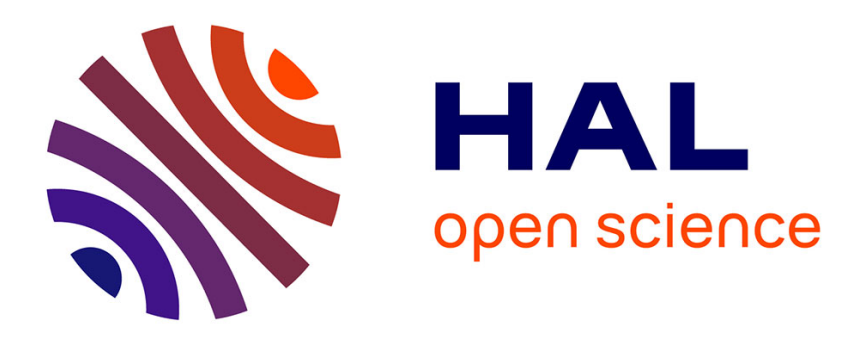

\title{
Randomized Quasi-Monte Carlo for Quantile Estimation
} Zachary T Kaplan, Yajuan Li, Marvin K Nakayama, Bruno Tuffin

\section{To cite this version:}

Zachary T Kaplan, Yajuan Li, Marvin K Nakayama, Bruno Tuffin. Randomized Quasi-Monte Carlo for Quantile Estimation. WSC 2019 - Winter Simulation Conference, Dec 2019, National Harbor, United States. pp.1-14. hal-02155421

\section{HAL Id: hal-02155421 \\ https://hal.inria.fr/hal-02155421}

Submitted on 13 Jun 2019

HAL is a multi-disciplinary open access archive for the deposit and dissemination of scientific research documents, whether they are published or not. The documents may come from teaching and research institutions in France or abroad, or from public or private research centers.
L'archive ouverte pluridisciplinaire $\mathbf{H A L}$, est destinée au dépôt et à la diffusion de documents scientifiques de niveau recherche, publiés ou non, émanant des établissements d'enseignement et de recherche français ou étrangers, des laboratoires publics ou privés. 


\title{
Randomized Quasi-Monte Carlo for Quantile Estimation
}

\author{
Zachary T. Kaplan \\ Yajuan Li \\ Marvin K. Nakayama \\ Computer Science Department \\ New Jersey Institute of Technology \\ Newark, NJ 07102, USA
}

\author{
Bruno Tuffin \\ Inria, Univ Rennes, CNRS, IRISA \\ Campus de Beaulieu \\ 35042 Rennes, FRANCE
}

\begin{abstract}
We compare two approaches for quantile estimation via randomized quasi-Monte Carlo (RQMC) in an asymptotic setting where the number of randomizations for RQMC grows large but the size of the low-discrepancy point set remains fixed. In the first method, for each randomization, we compute an estimator of the cumulative distribution function (CDF), which is inverted to obtain a quantile estimator, and the overall quantile estimator is the sample average of the quantile estimators across randomizations. The second approach instead computes a single quantile estimator by inverting one CDF estimator across all randomizations. Because quantile estimators are generally biased, the first method leads to an estimator that does not converge to the true quantile as the number of randomizations goes to infinity. In contrast, the second estimator does, and we establish a central limit theorem for it. Numerical results further illustrate these points.
\end{abstract}

\section{INTRODUCTION}

For a continuous random variable $Y$ with cumulative distribution function (CDF) $F$, the $p$-quantile $(0<$ $p<1)$ is the smallest constant $\xi$ such that $P(Y \leq \xi)=p$; i.e., $\xi=F^{-1}(p)$. For example, the median is the 0.5-quantile, also known as the 50th percentile. Many application areas employ quantiles to measure risk. In finance, a quantile is called a value-at-risk (e.g., see [11]), which is often used to specify appropriate capital levels. Nuclear engineers employ a 0.95-quantile in probabilistic safety assessments (PSAs) of nuclear power plants. When a PSA is performed using Monte Carlo (MC), the U.S. Nuclear Regulatory Commission (NRC) requires accounting for the resulting sampling error; e.g., see [32], Section 3.2 of [31], and Section 24.9 of [33]. This can be accomplished by providing a confidence interval (CI) for $\xi$.

The typical MC approach to estimate $\xi$ first estimates the CDF $F$, and then inverts the estimated CDF to obtain a quantile estimator; e.g., see Section 2.3 of [25]. Suppose a response $Y$ can be generated from $d \geq 1$ independent and identically distributed (i.i.d.) uniforms on $[0,1)$. Then for a specified sample size $n \geq 1$, we can form an MC estimator of $F$ by drawing a random sample of $n$ independent uniformly distributed points from the unit hypercube $[0,1)^{d}$; transforming each uniform vector into an observation of the response $Y$; and computing the empirical distribution function of the $n$ values of $Y$.

In contrast, quasi-Monte Carlo (QMC) evaluates the response function at a deterministic set of $n$ points that are carefully placed so that they more evenly cover $[0,1)^{d}$ than a typical random sample; see 
[19] and [15] for overviews of QMC. A QMC estimator of a mean can have a faster rate of convergence than the corresponding MC estimator. But providing explicit error bounds for a QMC estimator can be quite difficult.

Randomized QMC (RQMC) provides a way of producing computable error bounds. RQMC randomizes a QMC point set $r \geq 2$ independent times, where an estimator is computed from each randomization. Taking the sample mean and sample variance across the $r$ randomizations, one can then compute a CI. RQMC has been implemented in various ways, including random shift modulo 1 [7, 28], random digital shift [16], and scrambling [20]. Previous work on applying QMC or RQMC for quantile estimation includes [24], [10], [13], and [9], but these works do not consider the problem of constructing explicit error bounds, as one wants for a nuclear PSA.

For an RQMC estimator of a mean when using a digital net with full nested scrambling [20, 22, 21, 23], paper [17] establishes a central limit theorem (CLT) with a normally distributed limit as the size $m$ of the point set grows large, but the nested scrambling can be computationally costly. For other ways of implementing RQMC, as $m$ gets large, an estimator computed from a randomization of the point set may not satisfy a CLT with a Gaussian limit. For example, for estimators based on randomly shifting (modulo 1) a lattice, [14] analyze the limiting distribution, which they generally find to be nonnormal, and their Figures 15 and 19 show histograms displaying distinct asymmetry and/or multiple modes. Thus, for a CI based on RQMC to be asymptotically valid, we may need the number $r$ of randomizations to grow to infinity, which is the large-sample setting we now consider. In RQMC practice, though, it is common to choose $r$ as not too large, e.g., $r=30$, motivated by a common rule of thumb for when the asymptotics of a CLT roughly start holding.

Our paper examines asymptotic properties of two RQMC estimators of a quantile, where $r$ grows large but the size $m$ of the low-discrepancy point set remains fixed. In one approach, for each randomized point set, we compute a CDF estimator, which is inverted to obtain a quantile estimator. We then compute the sample average of the quantile estimators across the $r$ randomizations to obtain the final quantile estimator. Because quantile estimators are generally biased, this estimator does not converge to the true quantile in our asymptotic regime, which keeps $m$ fixed. This is in contrast to the corresponding RQMC estimator of a mean, which does converge to the true mean in this large-sample framework.

Our second quantile estimator instead computes a single CDF estimator using the responses from all randomizations, and then inverts the overall CDF estimator to obtain a single quantile estimator. We show that this RQMC quantile estimator, even though it is biased for fixed $r$ and $m$, does converge to the true quantile as $r$ grows large with $m$ fixed. This RQMC quantile estimator also satisfies a [3] representation and a CLT with a Gaussian limit. We further provide numerical results comparing the two RQMC quantile estimators, along with MC estimators, as either $r$ or $m$ grows large, with the other fixed.

The rest of our paper unfolds as follows. Section 2 describes the basic mathematical problem. In Sections 3 and 4, we review how to estimate a quantile using MC and QMC, respectively. Sections 5 and 6 develop our two RQMC quantile estimators. We provide numerical results in Section 7, and give some concluding remarks in Section 8. Due to space limitations, all proofs will appear in a follow-up paper. 


\section{MATHEMATICAL BACKGROUND}

For a given (deterministic) function $w_{Y}:[0,1)^{d} \rightarrow \Re$ with fixed integer $d \geq 1$, let

$$
Y=w_{Y}\left(U_{1}, U_{2}, \ldots, U_{d}\right)=w_{Y}(\mathbf{U})
$$

where $U_{1}, U_{2}, \ldots, U_{d}$, are i.i.d. $U[0,1)$ (i.e., uniform on the interval $[0,1)$ ) random variables, so $\mathbf{U}=$ $\left(U_{1}, U_{2}, \ldots, U_{d}\right) \sim U[0,1)^{d}$. We call $w_{Y}$ a response function, which may represent a simulation program that produces a response $Y$ using $d$ i.i.d. uniforms as input. The function $w_{Y}$ can be quite complicated, first converting $\mathbf{U} \sim U[0,1)^{d}$ into a random vector $\mathbf{Z}$ with non-identically distributed components having a dependence structure, and then performing computations using $\mathbf{Z}$, to finally produce $Y$. Let $F$ be the $\mathrm{CDF}$ of $Y$, which we assume cannot be computed analytically nor numerically. For each $y \in \mathfrak{R}$, we have that

$$
F(y)=P(Y \leq y)=P\left(w_{Y}(\mathbf{U}) \leq y\right)=\int_{[0,1)^{d}} I\left(w_{Y}(\mathbf{u}) \leq y\right) \mathrm{d} \mathbf{u},
$$

where $I(\cdot)$ denotes the indicator function, which equals 1 (resp., 0) when its argument is true (resp., false).

For a fixed value $0<p<1$, define

$$
\xi \equiv \xi_{p}=F^{-1}(p) \equiv \inf \{y: F(y) \geq p\},
$$

which is the $p$-quantile of $F$ (equivalently, of $Y$ ). Thus, in the case that $F$ is continuous, exactly $p$ of the mass of $F$ lies below $\xi$. Let $f$ denote the derivative (when it exists) of $F$, and we will assume throughout that $f(\xi)>0$, which ensures that $y=\xi$ is the unique solution of the equation $F(y)=p$.

The goal is to estimate $\xi$ using some form of Monte Carlo or quasi-Monte Carlo. The general approach we will follow to estimate $\xi=F^{-1}(p)$ is to first estimate the CDF $F$ and then invert the estimated CDF to obtain a quantile estimator. We further want to provide a measure of the error of our quantile estimator.

We next motivate the problem and illustrate the notation in the following example.

Example 1 Consider a system experiencing a random load $L$ with a random capacity $C$ to withstand the load. The system fails when $L \geq C$, so $Y \equiv C-L$ is the system's safety margin, which has CDF $F$. An example is a nuclear power plant undergoing a hypothesized accident, as studied in [8] and [26], where $L$ denotes the peak cladding temperature (PCT) during the postulated accident and $C$ is the temperature at which the cladding material suffers damage. It is reasonable to consider the PCT as a random variable because it depends on unforeseen aspects of the events (e.g., time and size of a pipe break) during the accident, and the capacity $C$ may be unknown because of the variability of the cladding's material properties, which are modeled as random variables.

In (2), we can think of the function $w_{Y}$ as follows. It first takes $d$ i.i.d. uniforms as input, transforming them into an observation of $(L, C)$, possibly with some dependence structure. Then $w_{Y}$ outputs $Y=C-L$.

Let $\theta=P(Y \leq 0)$, which is the failure probability, and a regulator may specify that $\theta$ must be less than a given threshold $\theta_{0}$, e.g., $\theta_{0}=0.05$. The requirement that $\theta<\theta_{0}$ can be equivalently reformulated in terms of a quantile: the $\theta_{0}$-quantile $\xi$ of $Y$ must satisfy $\xi>0$. 


\section{MONTE CARLO}

We now describe how to apply MC to estimate $\xi$. Fix a sample size $n \geq 2$, and generate a sample of $n$ independent random vectors $\mathbf{U}_{i}, i=1,2, \ldots, n$, where each $\mathbf{U}_{i}=\left(U_{i, 1}, U_{i, 2}, \ldots, U_{i, d}\right) \sim U[0,1)^{d}$. For each $i=1,2, \ldots, n$, define $Y_{i}=w_{Y}\left(\mathbf{U}_{i}\right)$, so $Y_{1}, Y_{2}, \ldots, Y_{n}$ is a sample of $n$ i.i.d. copies of $Y$, with each $Y_{i} \sim F$ by (1). Then we define the MC estimator of $F$ as the empirical distribution function

$$
\widehat{F}_{\mathrm{MC}, n}(y)=\frac{1}{n} \sum_{i=1}^{n} I\left(Y_{i} \leq y\right) \text {. }
$$

A natural estimator of $\xi=F^{-1}(p)$ is the $M C$ quantile estimator

$$
\widehat{\xi}_{\mathrm{MC}, n}=\widehat{F}_{\mathrm{MC}, n}^{-1}(p),
$$

which can be computed through order statistics. Specifically, let $Y_{1: n} \leq Y_{2: n} \leq \cdots \leq Y_{n: n}$ be the ordered values of the sample $Y_{1}, Y_{2}, \ldots, Y_{n}$. Let $\lceil\cdot\rceil$ denote the ceiling function, and we have that

$$
\widehat{\xi}_{\mathrm{MC}, n}=Y_{\lceil n p\rceil: n} .
$$

\subsection{Large-Sample Properties of MC Quantile Estimator}

A Bahadur representation [3], described next, provides a useful approach for analyzing the large-sample properties of $\widehat{\xi}_{\mathrm{MC}, n}$. For $n$ sufficiently large, there exists a neighborhood $\mathscr{N}_{n}$ of $\xi$ such that

$$
\widehat{F}_{\mathrm{MC}, n}(y) \approx \widehat{F}_{\mathrm{MC}, n}(\xi)+F(y)-F(\xi) \text { uniformly for } y \text { in } \mathscr{N}_{n},
$$

and $\mathscr{N}_{n}$ contains $\widehat{\xi}_{\mathrm{MC}, n}$ with probability 1 . Thus, because $\widehat{\xi}_{\mathrm{MC}, n}=\widehat{F}_{\mathrm{MC}, n}^{-1}(p)$, we have that

$$
p \approx \widehat{F}_{\mathrm{MC}, n}\left(\widehat{\xi}_{\mathrm{MC}, n}\right) \approx \widehat{F}_{\mathrm{MC}, n}(\xi)+F\left(\widehat{\xi}_{\mathrm{MC}, n}\right)-F(\xi) \approx \widehat{F}_{\mathrm{MC}, n}(\xi)+f(\xi)\left(\widehat{\xi}_{\mathrm{MC}, n}-\xi\right),
$$

where the last step follows from a first-order Taylor approximation. Under our assumption from Section 2 that $f(\xi)>0$, rearranging terms leads to

$$
\widehat{\xi}_{\mathrm{MC}, n} \approx \xi+\frac{p-\widehat{F}_{\mathrm{MC}, n}(\xi)}{f(\xi)},
$$

so the quantile estimator roughly equals the true quantile plus a linear transformation of a CDF estimator evaluated at $\xi$.

[3] formalizes the above discussion. Specifically, if $f(\xi)>0$, then

$$
\begin{gathered}
\widehat{\xi}_{\mathrm{MC}, n}=\xi+\frac{p-\widehat{F}_{\mathrm{MC}, n}(\xi)}{f(\xi)}+R_{n}, \\
\text { with } \sqrt{n} R_{n} \Rightarrow 0 \text { as } n \rightarrow \infty,
\end{gathered}
$$

where $\Rightarrow$ denotes convergence in distribution (e.g., Section 25 of [4]). We call (6)-(7) a (weak) Bahadur representation. Under the additional assumption that $F$ is twice differentiable at $\xi$, [3] actually proves a 
stronger result than (7), namely that $R_{n}$ vanishes at rate $O\left(n^{-3 / 4} \log n\right)$ almost surely (a.s.); see Section 2.5 of [25] for refinements.

The Bahadur representation implies that the MC quantile estimator satisfies a CLT. From (6), we have

$$
\sqrt{n}\left[\widehat{\xi}_{\mathrm{MC}, n}-\xi\right]=\frac{\sqrt{n}}{f(\xi)}\left[p-\widehat{F}_{\mathrm{MC}, n}(\xi)\right]+\sqrt{n} R_{n}
$$

By (3), $\widehat{F}_{\mathrm{MC}, n}(\xi)$ averages i.i.d. copies of $I(Y \leq \xi)$, which has mean $E[I(Y \leq \xi)]=p$ and variance $\psi_{\mathrm{MC}}^{2} \equiv \operatorname{Var}[I(Y \leq \xi)]=p(1-p)$. Thus, the ordinary $C L T$ (e.g., Theorem 27.1 of [4]) ensures

$$
\sqrt{n}\left[p-\widehat{F}_{\mathrm{MC}, n}(\xi)\right] \Rightarrow N\left(0, \psi_{\mathrm{MC}}^{2}\right) \quad \text { as } n \rightarrow \infty,
$$

where $N\left(a, b^{2}\right)$ is a normal random variable with mean $a$ and variance $b^{2}$. Hence, using (9) and (7) in (8), and applying Slutsky's theorem (e.g., p. 19 of [25]), we get that

$$
\sqrt{n}\left[\widehat{\xi}_{\mathrm{MC}, n}-\xi\right] \Rightarrow \frac{1}{f(\xi)} N\left(0, \psi_{\mathrm{MC}}^{2}\right)+0 \stackrel{\mathscr{D}}{=} N\left(0, \tau_{\mathrm{MC}}^{2}\right) \quad \text { as } n \rightarrow \infty,
$$

where $\stackrel{\mathscr{D}}{=}$ denotes equality in distribution, and

$$
\tau_{\mathrm{MC}}^{2} \equiv \frac{\psi_{\mathrm{MC}}^{2}}{f^{2}(\xi)}=\frac{p(1-p)}{f^{2}(\xi)} .
$$

Therefore, even though $\widehat{\xi}_{\mathrm{MC}, n}$ is not a sample average, it still obeys a CLT because the Bahadur representation shows that the large-sample asymptotics of $\widehat{\xi}_{\mathrm{MC}, n}$ can be well-approximated by those of $\widehat{F}_{\mathrm{MC}, n}(\xi)$, which is a sample average satisfying the CLT in (9).

One measure of the error of a Monte Carlo estimator is its (root) mean squared error ((R)MSE). For our MC quantile estimator $\widehat{\xi}_{\mathrm{MC}, n}$ in (4), Theorem 2 of [2] shows that

$$
\operatorname{MSE}\left[\widehat{\xi}_{\mathrm{MC}, n}\right]=E\left[\left(\widehat{\xi}_{\mathrm{MC}, n}-\xi\right)^{2}\right]=n^{-1} \tau_{\mathrm{MC}}^{2}+o\left(n^{-1}\right)=O\left(n^{-1}\right)
$$

as $n \rightarrow \infty$, where for two functions $g_{1}(n)$ and $g_{2}(n)$, we write that $g_{1}(n)=O\left(g_{2}(n)\right)$ as $n \rightarrow \infty$ if there exists a constant $c$ such that $\left|g_{1}(n)\right| \leq c\left|g_{2}(n)\right|$ for all $n$ sufficiently large, and $g_{1}(n)=o\left(g_{2}(n)\right)$ means that $g_{1}(n) / g_{2}(n) \rightarrow 0$ as $n \rightarrow \infty$. Thus, although $\widehat{\xi}_{\mathrm{MC}, n}$ is generally biased, its MSE is dominated by its asymptotic variance $\tau_{\mathrm{MC}}^{2}$ from (11); also see Lemma 1 of [2]. We then see that

$$
\operatorname{RMSE}\left[\widehat{\xi}_{\mathrm{MC}, n}\right]=n^{-1 / 2} \tau_{\mathrm{MC}}+o\left(n^{-1 / 2}\right)=O\left(n^{-1 / 2}\right)
$$

as $n \rightarrow \infty$, which provides a measure of the rate of convergence of the MC quantile estimator.

Another way of describing the error in $\widehat{\xi}_{\mathrm{MC}, n}$ is through a confidence interval. We can unfold the CLT (10) to obtain an asymptotic $\beta$-level $(0<\beta<1)$ two-sided CI for $\xi$ as

$$
J_{\mathrm{MC}, n}^{\prime} \equiv\left[\widehat{\xi}_{\mathrm{MC}, n} \pm z_{1-(1-\beta) / 2} \tau_{\mathrm{MC}} / \sqrt{n}\right]
$$


where $z_{q}=\Phi^{-1}(q)$ for $0<q<1$ and $\Phi$ is the $N(0,1) \operatorname{CDF}$ (e.g., $\left.z_{0.95}=1.96\right)$. However, this $\mathrm{CI}$ is not directly implementable because $f(\xi)$ in $\tau_{\mathrm{MC}}^{2}$ of (11) is typically unknown. But it is possible (e.g., see [5]) to construct a consistent estimator $\widehat{\tau}_{\mathrm{MC}, n}^{2}$ of $\tau_{\mathrm{MC}}^{2}$; i.e., $\widehat{\tau}_{\mathrm{MC}, n}^{2} \Rightarrow \tau_{\mathrm{MC}}^{2}$ as $n \rightarrow \infty$. We can then obtain a large-sample $\beta$-level two-sided CI for $\xi$ as

$$
J_{\mathrm{MC}, n} \equiv\left[\widehat{\xi}_{\mathrm{MC}, n} \pm z_{1-(1-\beta) / 2} \widehat{\tau}_{\mathrm{MC}, n} / \sqrt{n}\right],
$$

which is asymptotically valid in the sense that $\lim _{n \rightarrow \infty} P\left(\xi \in J_{\mathrm{MC}, n}\right)=\beta$, or equivalently,

$$
P\left(\left|\widehat{\xi}_{\mathrm{MC}, n}-\xi\right| \leq z_{1-(1-\beta) / 2} \widehat{\tau}_{\mathrm{MC}, n} / \sqrt{n}\right) \rightarrow \beta, \quad \text { as } n \rightarrow \infty .
$$

As a consequence, we have that $\left|\widehat{\xi}_{\mathrm{MC}, n}-\xi\right|=O_{p}\left(n^{-1 / 2}\right)$ as $n \rightarrow \infty$, where the notation $X_{n}=O_{p}\left(a_{n}\right)$ for a sequence of random variables $X_{n}, n \geq 1$, and constants $a_{n}, n \geq 1$, means that $X_{n} / a_{n}$ is bounded in probability (Section 1.2.5 of [25]).

\section{QUASI-MONTE CARLO}

Rather than estimating $\xi$ with random sampling as in Monte Carlo, QMC instead evaluates the response function at carefully placed deterministic points in $[0,1)^{d}$, which are chosen to be more evenly dispersed over $[0,1)^{d}$ than a typical random sample of i.i.d. uniforms. Let $\mathscr{P}_{n}=\left\{\mathbf{u}_{1}, \mathbf{u}_{2}, \ldots, \mathbf{u}_{n}\right\}$ be a low-discrepancy point set of size $n$, where each $\mathbf{u}_{i}=\left(u_{i, 1}, u_{i, 2}, \ldots, u_{i, d}\right) \in[0,1)^{d}$. Such a $\mathscr{P}_{n}$ can be constructed deterministically as a lattice [27] or a digital net, including ones designed by Halton, Faure, Sobol', and Niederreiter; see Chapters 3-5 of [19] or Chapter 5 of [15] for an overview.

The QMC estimator of $F(y)$ in (2) is

$$
\widehat{F}_{\mathrm{QMC}, n}(y)=\frac{1}{n} \sum_{i=1}^{n} I\left(w_{Y}\left(\mathbf{u}_{i}\right) \leq y\right) .
$$

We call $\widehat{F}_{\mathrm{QMC}, n}(\cdot)$ the QMC CDF estimator, and we invert $\widehat{F}_{\mathrm{QMC}, n}$ to obtain the QMC quantile estimator

$$
\widehat{\xi}_{\mathrm{QMC}, n}=\widehat{F}_{\mathrm{QMC}, n}^{-1}(p) \text {. }
$$

Just as for the MC $p$-quantile estimator in (5), we can compute $\widehat{\xi}_{\mathrm{QMC}, n}$ in (13) by sorting $w_{Y}\left(\mathbf{u}_{i}\right), i=$ $1,2, \ldots, n$, in ascending order, and setting $\widehat{\xi}_{\mathrm{QMC}, n}$ equal to the $\lceil n p\rceil$-th smallest one.

Recall that by (12), the RMSE of the MC quantile estimator converges at rate $O\left(n^{-1 / 2}\right)$, where $n$ is the sample size, and we would like to provide analogous (deterministic) error bounds for $\left|\widehat{\xi}_{\mathrm{QMC}, n}-\xi\right|$. One approach is to try the following. For the moment, suppose that we are interested in computing the integral

$$
\gamma \equiv \int_{[0,1)^{d}} h(\mathbf{u}) \mathrm{d} \mathbf{u}
$$

for some integrand $h:[0,1)^{d} \rightarrow \Re$, and we estimate $\gamma$ by the QMC estimator $(1 / n) \sum_{i=1}^{n} h\left(\mathbf{u}_{i}\right)$, with low-discrepancy point set $\mathscr{P}_{n}=\left\{\mathbf{u}_{1}, \mathbf{u}_{2}, \ldots, \mathbf{u}_{n}\right\}$. Then the Koksma-Hlawka inequality states that

$$
\left|\frac{1}{n} \sum_{i=1}^{n} h\left(\mathbf{u}_{i}\right)-\gamma\right| \leq D^{*}\left(\mathscr{P}_{n}\right) V_{\mathrm{HK}}(h),
$$


where $D^{*}\left(\mathscr{P}_{n}\right)$ is the star-discrepancy of $\mathscr{P}_{n}$, which is a measure of the uniformity of $\mathscr{P}_{n}$ over $[0,1)^{d}$, and $V_{\mathrm{HK}}(h)$ is the Hardy-Krause (HK) variation of the integrand $h$, specifying its roughness; see Section 5.6 of [15] for details. Low-discrepancy point sets $\mathscr{P}_{n}$ often have $D^{*}\left(\mathscr{P}_{n}\right)=O\left((\log n)^{v} / n\right)$ for some constant $v>0$ (e.g., $v=d-1$ or $v=d$ ) as $n \rightarrow \infty$. Hence, when the integrand $h$ is sufficiently smooth so that $V_{\mathrm{HK}}(h)<\infty,(15)$ implies that the deterministic rate at which the QMC integration error decreases is $O\left((\log n)^{v} / n\right)$ as $n \rightarrow \infty$, better than MC's rate of $O\left(n^{-1 / 2}\right)$.

But there are several problems with this approach of trying to bound the QMC error. When estimating the CDF $F(y)$ in (2), the integrand is $h_{y}(\mathbf{u})=I\left(w_{Y}(\mathbf{u}) \leq y\right)$, which is discontinuous in $\mathbf{u}$ and typically has $V_{\mathrm{HK}}\left(h_{y}\right)=\infty$, so the upper bound in the Koksma-Hlawka inequality (15) is infinite. Even if the HK variation of the integrand $h$ were finite, computing the bound in (15) is at least as difficult as computing the integral in (14), and the bound can be quite conservative, making (15) impractical. Moreover, the bound in (15) for integrand $h_{y}$ is for the QMC CDF estimator at $y$, not for $\left|\widehat{\xi}_{\mathrm{QMC}, n}-\xi\right|$, which is what we are actually interested in.

\section{ONE APPROACH OF RANDOMIZED QUASI-MONTE CARLO}

Rather than trying to provide a deterministic error bound for the QMC quantile estimator, we can instead attempt to use RQMC to obtain a CI for $\xi$. For a given low-discrepancy point set, the basic idea is to randomize the set $r \geq 2$ independent times in a way that retains the low-discrepancy property for each randomization, and compute an estimator from each of the $r$ independent randomizations. Then we can form a CI from the sample mean and sample variance across randomizations. For a fair comparison to the $p$-quantile estimator using MC in (4) or via QMC in (13), each of which is based on $n$ evaluations of the response function $w_{Y}$ in (1), we also want to apply RQMC using the same total number $n$ of function evaluations. We next describe details on how RQMC may be implemented.

Let $r \geq 2$ be the number of randomizations to use for RQMC, and let $\mathscr{P}_{m}=\left\{\mathbf{u}_{1}, \mathbf{u}_{2}, \ldots, \mathbf{u}_{m}\right\}$ be a low-discrepancy point set of size $m=n / r$, where each $\mathbf{u}_{i} \in[0,1)^{d}$, and we assume that $n / r$ is an integer. For each $k=1,2, \ldots, r$, we want to perform a randomization of $\mathscr{P}_{m}$ to obtain another point set

$$
\mathscr{P}_{m}^{(k)}=\left\{\mathbf{X}_{1}^{(k)}, \mathbf{X}_{2}^{(k)}, \ldots, \mathbf{X}_{m}^{(k)}\right\}
$$

with each $\mathbf{X}_{i}^{(k)}=\left(X_{i, 1}^{(k)}, X_{i, 2}^{(k)}, \ldots X_{i, d}^{(k)}\right)$, such that

$$
\mathbf{X}_{i}^{(k)} \sim U[0,1)^{d}, \text { for each } i=1,2, \ldots, m, \text { and each } k=1,2, \ldots, r,
$$

and

$$
\mathscr{P}_{m}^{(1)}, \mathscr{P}_{m}^{(2)}, \ldots, \mathscr{P}_{m}^{(r)} \text { are i.i.d. }
$$

One simple way of constructing $\mathscr{P}_{m}^{(k)}$ in (16) satisfying (17) and (18) is through independent random shifts. First generate $\mathbf{S}_{1}, \mathbf{S}_{2}, \ldots, \mathbf{S}_{r}$ as $r$ independent random vectors, where each

$$
\mathbf{S}_{k}=\left(S_{k, 1}, S_{k, 2}, \ldots, S_{k, d}\right) \sim U[0,1)^{d} .
$$

For each $k=1,2, \ldots, r$, we will shift (modulo 1) each point in the original set $\mathscr{P}_{m}$ by $\mathbf{S}_{k}$ to obtain $\mathscr{P}_{m}^{(k)}$. Specifically, for $\mathbf{x}=\left(x_{1}, x_{2}, \ldots, x_{d}\right) \in \mathfrak{R}^{d}$ and $\mathbf{y}=\left(y_{1}, y_{2}, \ldots, y_{d}\right) \in \mathfrak{R}^{d}$, define the operator $\oplus$ as

$$
\mathbf{x} \oplus \mathbf{y}=\left(\left(x_{1}+y_{1}\right) \bmod 1,\left(x_{2}+y_{2}\right) \bmod 1, \ldots,\left(x_{d}+y_{d}\right) \bmod 1\right) .
$$


For each $k=1,2, \ldots, r$, we then obtain a shifted point set $\mathscr{P}_{m}^{(k)}$ in (16) with each $\mathbf{X}_{i}^{(k)}=\mathbf{u}_{i} \oplus \mathbf{S}_{k}$; i.e., each point in the original point set is shifted by the same random uniform $\mathbf{S}_{k}$. It is easy to show that each $\mathbf{u}_{i} \oplus \mathbf{S}_{k} \sim U[0,1)^{d}$, so (17) holds. Each shifted point set $\mathscr{P}_{m}^{(k)}$ uses the same low-discrepancy point set $\mathscr{P}_{m}$ but a different random shift $\mathbf{S}_{k}$. The $m$ points in any $\mathscr{P}_{m}^{(k)}$ will be stochastically dependent because they all share the same random shift $\mathbf{S}_{k}$. But the $r$ shifted point sets $\mathscr{P}_{m}^{(k)}, k=1,2, \ldots, r$, are stochastically independent because $\mathbf{S}_{k}, k=1,2, \ldots, r$, are independent, thereby implying (18).

When the original point set $\mathscr{P}_{m}$ is a lattice, each shifted point set $\mathscr{P}_{m}^{(k)}$ retains a lattice structure. But if $\mathscr{P}_{m}$ is a digital net, its random shift $\mathscr{P}_{m}^{(k)}$ may no longer be a digital net. In this latter case, we instead can apply scrambling to obtain each $\mathscr{P}_{m}^{(k)}$ satisfying (17) and (18), where the scrambled point set still possesses the desirable properties of the original point set; see [21, 22].

In whatever way we obtain the $r$ randomized point sets $\mathscr{P}_{m}^{(1)}, \mathscr{P}_{m}^{(2)}, \ldots, \mathscr{P}_{m}^{(r)}$ satisfying (17) and (18), for each randomization $k=1,2, \ldots, r$, let

$$
\widehat{F}_{\mathrm{RQMC}, m, k}(y)=\frac{1}{m} \sum_{i=1}^{m} I\left(w_{Y}\left(\mathbf{X}_{i}^{(k)}\right) \leq y\right),
$$

which is a CDF estimator computed from the randomized point set $\mathscr{P}_{m}^{(k)}$. Even though $\mathbf{X}_{i}^{(k)}, i=$ $1,2, \ldots, m$, are stochastically dependent, we still have that $E\left[\widehat{F}_{\mathrm{RQMC}, m, k}(y)\right]=F(y)$ for each $y$ by (17). We invert $\widehat{F}_{\mathrm{RQMC}, m, k}$ to obtain

$$
\widehat{\xi}_{\mathrm{RQMC}, m, k}=\widehat{F}_{\mathrm{RQMC}, m, k}^{-1}(p),
$$

where $\widehat{\xi}_{\mathrm{RQMC}, m, k}, k=1,2, \ldots, r$, are i.i.d. Then an RQMC estimator of $\xi$ is

$$
\bar{\xi}_{\mathrm{RQMC}, m, r}=\frac{1}{r} \sum_{k=1}^{r} \widehat{\xi}_{\mathrm{RQMC}, m, k} .
$$

As noted previously at the end of Section 4, when estimating the CDF in (2), the integrand $I\left(w_{Y}(\mathbf{u}) \leq\right.$ $y$ ) typically has infinite HK variation, making theoretical bounds of the form in (15) not useful. But when instead estimating the integral in (14) with an integrand $h(\mathbf{u})$ having finite HK variation, applying RQMC leads to certain benefits over MC and QMC. First, for the estimator from each randomization in RQMC, the rate at which its variance decreases is $O\left(m^{-2}(\log m)^{d}\right)$ as $m$ grows, and even faster in some cases, as detailed in $[16,22,29]$. In contrast, the $\mathrm{MC}$ variance of a sample of size $m$ decreases at rate $O\left(\mathrm{~m}^{-1}\right)$. Compared to QMC, RQMC allows for practical estimation of the approximation error through a CLT. Moreover, even when the HK variation is infinite, making inequalities such as (15) uninformative, numerical experiments show that the empirical behavior of RQMC's convergence rate can be substantially better than that of MC [30].

\subsection{Large-Sample Properties of RQMC Quantile Estimator in (20)}

The RQMC quantile estimator $\bar{\xi}_{\mathrm{RQMC}, m, r}$ in (20) satisfies the following CLT, where $\left(\widehat{F}_{\mathrm{RQMC}, m}, \widehat{\xi}_{\mathrm{RQMC}, m}\right)$ denotes a generic copy of $\left(\widehat{F}_{\mathrm{RQMC}, m, k}, \widehat{\xi}_{\mathrm{RQMC}, m, k}\right)$.

Proposition 1 If $\tau_{\mathrm{RQMC}, m}^{\prime 2} \equiv \operatorname{Var}\left[\widehat{\xi}_{\mathrm{RQMC}, m}\right]<\infty$, then for fixed $m \geq 1$,

$$
\sqrt{r}\left(\bar{\xi}_{\mathrm{RQMC}, m, r}-E\left[\widehat{\xi}_{\mathrm{RQMC}, m}\right]\right) \Rightarrow N\left(0, \tau_{\mathrm{RQMC}, m}^{\prime 2}\right) \quad \text { as } r \rightarrow \infty .
$$


It is important to note that the centering constant on the left-hand side of CLT (21) is not the true $p$-quantile $\xi$ but rather $E\left[\widehat{\xi}_{\mathrm{RQMC}, m}\right]$. While $\widehat{F}_{\mathrm{RQMC}, m}(y)$ is an unbiased estimator of $F(y)$ for each $m$ and $y$, its inverse $\widehat{\xi}_{\mathrm{RQMC}, m}=\widehat{F}_{\mathrm{RQMC}, m}^{-1}(p)$ is typically a biased estimator of $\xi$ because of the nonlinearity of the inversion operation. Because $\bar{\xi}_{\mathrm{RQMC}, m, r}$ averages $r$ i.i.d. copies of $\widehat{\xi}_{\mathrm{RQMC}, m}$, we see that

$$
E\left[\bar{\xi}_{\mathrm{RQMC}, m, r}\right]=E\left[\widehat{\xi}_{\mathrm{RQMC}, m}\right] \neq \xi
$$

in general for fixed $m$. In addition, for fixed $m$, we have that a.s.,

$$
\lim _{r \rightarrow \infty} \bar{\xi}_{\mathrm{RQMC}, m, r}=E\left[\widehat{\xi}_{\mathrm{RQMC}, m}\right] \neq \xi
$$

by the strong law of large numbers, so $\bar{\xi}_{\mathrm{RQMC}, m, r}$ converges to the wrong value as $r \rightarrow \infty$ for fixed $m$.

Because $\operatorname{Bias}\left[\bar{\xi}_{\mathrm{RQMC}, m, r}\right]=\operatorname{Bias}\left[\widehat{\xi}_{\mathrm{RQMC}, m}\right]=E\left[\widehat{\xi}_{\mathrm{RQMC}, m}\right]-\xi$, we have that

$$
\begin{aligned}
\operatorname{MSE}\left[\bar{\xi}_{\mathrm{RQMC}, m, r}\right] & =\left(\operatorname{Bias}\left[\bar{\xi}_{\mathrm{RQMC}, m, r}\right]\right)^{2}+\operatorname{Var}\left[\bar{\xi}_{\mathrm{RQMC}, m, r}\right] \\
& =\left(\operatorname{Bias}\left[\widehat{\xi}_{\mathrm{RQMC}, m}\right]\right)^{2}+\frac{1}{r} \operatorname{Var}\left[\widehat{\xi}_{\mathrm{RQMC}, m}\right]
\end{aligned}
$$

Although the second term in (24) decreases at rate $r^{-1}$ as $r \rightarrow \infty$, (22) implies that the first is nonzero and does not shrink for fixed $m$, so

$$
\operatorname{RMSE}\left[\bar{\xi}_{\mathrm{RQMC}, m, r}\right]=\operatorname{Bias}\left[\widehat{\xi}_{\mathrm{RQMC}, m}\right]+o(1)=O(1)
$$

as $r \rightarrow \infty$ with $m$ fixed. Thus, the RMSE of $\bar{\xi}_{\mathrm{RQMC}, m, r}$ does not converge to 0 as $r \rightarrow \infty$ for fixed $m$.

Moreover, suppose we unfold the CLT (21) to build a $\beta$-level CI for $\xi$ as

$$
J_{\mathrm{RQMC}, m, r}=\left(\bar{\xi}_{\mathrm{RQMC}, m, r} \pm z_{1-(1-\beta) / 2} \widehat{\tau}_{\mathrm{RQMC}, m, r}^{\prime} / \sqrt{r}\right),
$$

where $\left.\widehat{\tau}_{\mathrm{RQMC}, m, r}^{\prime 2}=(1 /(r-1)) \sum_{k=1}^{r}\left[\widehat{\xi}_{\mathrm{RQMC}, m, k}\right)-\bar{\xi}_{\mathrm{RQMC}, m, r}\right]^{2}$ is a consistent estimator of $\tau_{\mathrm{RQMC}, m}^{\prime 2}$. Because the midpoint of $J_{\mathrm{RQMC}, m, r}$ is the biased estimator $\bar{\xi}_{\mathrm{RQMC}, m, r}$, the CI is centered at the wrong point on average, which can lead to poor coverage as $r \rightarrow \infty$ with $m$ fixed. We can try to address this issue by also letting $m \rightarrow \infty$, but we would then need to determine the relative rates at which $m \rightarrow \infty$ and $r \rightarrow \infty$ to ensure a CLT still holds.

\section{ANOTHER APPROACH OF RQMC FOR QUANTILE ESTIMATION}

As we explained in Section 5.1, the RQMC quantile estimator $\bar{\xi}_{\mathrm{RQMC}, m, r}$ in (20) does not converge to $\xi$ as $r \rightarrow \infty$ for fixed $m$. We next consider another RQMC estimator that, although biased, does converge in this setting.

Rather than compute a quantile estimator from each of the $r$ randomizations, as in (19), we instead construct a single overall CDF estimator from all $r$ randomizations, and then invert this to obtain a single 
overall quantile estimator. Specifically, first define the CDF estimator based on all $\mathrm{rm}$ evaluations of the response function $w_{Y}$ as

$$
\widetilde{F}_{\mathrm{RQMC}, m, r}(y)=\frac{1}{r} \sum_{k=1}^{r} \widehat{F}_{\mathrm{RQMC}, m, k}(y)=\frac{1}{r m} \sum_{k=1}^{r} \sum_{i=1}^{m} I\left(w_{Y}\left(\mathbf{X}_{i}^{(k)}\right) \leq y\right),
$$

which we call the overall CDF estimator. We then invert this to obtain another RQMC quantile estimator

$$
\widetilde{\xi}_{\mathrm{RQMC}, m, r}=\widetilde{F}_{\mathrm{RQMC}, m, r}^{-1}(p) .
$$

\subsection{Large-Sample Properties of RQMC Quantile Estimator in (28)}

Because $\widehat{F}_{\mathrm{RQMC}, m, k}, k=1,2, \ldots, r$, are i.i.d., with each $0 \leq \widehat{F}_{\mathrm{RQMC}, m, k}(y) \leq 1$ for all $y$, we have that the overall CDF estimator $\widetilde{F}_{\mathrm{RQMC}, m, r}$ in (27) at $\xi$ satisfies a CLT

$$
\sqrt{r}\left[\widetilde{F}_{\mathrm{RQMC}, m, r}(\xi)-p\right] \Rightarrow N\left(0, \psi_{\mathrm{RQMC}, m}^{2}\right) \quad \text { as } r \rightarrow \infty \text {, with } m \text { fixed. }
$$

By applying the theoretical framework developed in [6], we can establish the following properties of the corresponding quantile estimator $\widetilde{\xi}_{\mathrm{RQMC}, m, r}$ in (28).

Theorem 1 If $f(\xi)>0$, then for any fixed $m>0$,

$$
\begin{gathered}
\widetilde{\xi}_{\mathrm{RQMC}, m, r}=\xi+\frac{p-\widetilde{F}_{\mathrm{RQMC}, m, r}(\xi)}{f(\xi)}+R_{r}^{\prime}, \\
\text { with } \sqrt{r} R_{r}^{\prime} \Rightarrow 0, \quad \text { as } r \rightarrow \infty .
\end{gathered}
$$

Moreover, for each fixed $m>0$,

$$
\sqrt{r}\left[\widetilde{\xi}_{\mathrm{RQMC}, m, r}-\xi\right] \Rightarrow N\left(0, \tau_{\mathrm{RQMC}, m}^{2}\right) \quad \text { as } r \rightarrow \infty,
$$

where $\tau_{\mathrm{RQMC}, m}^{2}=\psi_{\mathrm{RQMC}, m}^{2} / f^{2}(\xi)$ for $\psi_{\mathrm{RQMC}, m}^{2}$ in (29). If in addition $\left\{r\left(\widetilde{\xi}_{\mathrm{RQMC}, m, r}-\xi\right)^{2}: r \geq 1\right\}$ is uniformly integrable (e.g., p. 338 of [4]), then

$$
\operatorname{RMSE}\left[\widetilde{\xi}_{\mathrm{RQMC}, m, r}\right]=r^{-1 / 2} \tau_{\mathrm{RQMC}, m}^{2}+o\left(r^{-1 / 2}\right)=O\left(r^{-1 / 2}\right)
$$

as $r \rightarrow \infty$ for fixed $m$.

Note that (30) and (31) establish a Bahadur representation for $\widetilde{\xi}_{\mathrm{RQMC}, m, r}$ as $r \rightarrow \infty$ with $m$ fixed. Also, even though $\widetilde{\xi}_{\mathrm{RQMC}, m, r}$ is biased for fixed $r$ and $m$, the CLT in (32) is centered at the true quantile $\xi$, in contrast to the CLT (21). Comparing (33) with (25), we see the advantage of the RQMC quantile estimator $\widetilde{\xi}_{\mathrm{RQMC}, m, r}$ in (28) over $\bar{\xi}_{\mathrm{RQMC}, m, r}$ in (20): as $r \rightarrow \infty$ with $m$ fixed, the RMSE of $\widetilde{\xi}_{\mathrm{RQMC}, m, r}$ shrinks to 0 but the RMSE of $\bar{\xi}_{\mathrm{RQMC}, m, r}$ does not. The RMSE of $\widetilde{\xi}_{\mathrm{RQMC}, m, r}$ converges at rate $r^{-1 / 2}$, which is the standard MC rate. But the numerical results in the next section show that RQMC can lead to substantially smaller MSE than MC, so we view RQMC as an MSE-reduction technique. 


\section{NUMERICAL RESULTS}

We now present results from running numerical experiments with the model in Example 1 from Section 2, which is motivated by studies of nuclear power plants undergoing hypothesized accidents; e.g., see $[8,26,1]$. The goal is to estimate the 0.05 -quantile $\xi$ of the safety margin $Y \sim F$, where $Y=C-L$. Let $G$ denote the joint CDF of $(L, C)$, and let $G_{L}$ and $G_{C}$ be the marginal CDFs of the load $L$ and the capacity $C$, respectively. As in $[8,26]$, we assume that $L$ and $C$ are independent, and we specify $G_{C}$ as triangular with support $[1800,2600]$ and mode 2200.

Reference [1] assumes that the load's marginal distribution $G_{L}$ is a mixture of $t=4$ lognormals, which we also use. Specifically, for each $s=1,2, \ldots, t$, let $G_{L,\langle s\rangle}$ be the CDF of $L_{\langle s\rangle}=\exp \left(\mu_{\langle s\rangle}+\sigma_{\langle s\rangle} Z_{\langle s\rangle}\right)$, where $Z_{\langle s\rangle} \sim N(0,1)$, and $\mu_{\langle s\rangle}$ and $\sigma_{\langle s\rangle}>0$ are given constants, so $L_{\langle s\rangle}$ has a lognormal distribution. Our experiments set $\mu_{\langle s\rangle}=7.4+0.1 s$ and $\sigma_{\langle s\rangle}=0.01+0.01 s$, which are as in [18]. Then define $G_{L}$ as a mixture of $G_{L,\langle s\rangle}, 1 \leq s \leq t$; i.e., $G_{L}(y)=\sum_{s=1}^{t} \lambda_{\langle s\rangle} G_{L,\langle s\rangle}(y)$ for given positive constants $\lambda_{\langle s\rangle}, 1 \leq s \leq t$, summing to 1 . We set $\lambda_{\langle 1\rangle}=0.99938 \times 0.9981 \times 0.919, \lambda_{\langle 2\rangle}=0.00062, \lambda_{\langle 3\rangle}=0.99938 \times 0.9981 \times 0.081$, and $\lambda_{\langle 4\rangle}=0.99938 \times 0.0019$, where the factors in each product match branching probabilities given in an event tree in Figure 2 of [8].

We can define the function $w_{Y}$ in (1) to take $d=3$ i.i.d. uniform inputs to generate $Y=w_{Y}\left(U_{1}, U_{2}, U_{3}\right)$. The function $w_{Y}$ uses $U_{1}$ and $U_{2}$ to generate the load $L \sim G_{L}$ as follows. First it employs $U_{1}$ to generate a discrete random variable $K$ with support $R=\{1,2, \ldots, t\}$ and probability mass function $P(K=s)=\lambda_{\langle s\rangle}$. If $K=s$, then generate $L$ having CDF $G_{L,\langle s\rangle}$, which is lognormal. Specifically, if $K=s$, let $L=\exp \left(\mu_{\langle s\rangle}+\right.$ $\left.\sigma_{\langle s\rangle} \Phi^{-1}\left(U_{2}\right)\right)$ where $\Phi$ is the $N(0,1)$ CDF. Also, $w_{Y}$ generates the capacity as $C=G_{C}^{-1}\left(U_{3}\right)$. Finally, $w_{Y}$ returns $Y=C-L$. Because of the analytical tractability of the model, we were able to numerically compute the 0.05 -quantile as $\xi=11.79948572$.

To examine the effect of the problem dimension $d$ on RQMC, we also considered another stochastically equivalent version of the model with larger $d$. Specifically, we artificially increase the dimension by generating the lognormal $L_{\langle s\rangle}$ as the exponential of a sum of $d^{\prime}=20$ independent normals with different marginal variances so that for each $s$, the sum of the $d^{\prime}$ marginal variances equals $\sigma_{\langle s\rangle}^{2}$. To specify the different marginal variances, we sampled $d^{\prime}$ independent chi-square random variables $V_{s, 1}, V_{s, 2}, \ldots, V_{s, d^{\prime}}$, and set the marginal variance of the $j$ th summand as $\sigma_{\langle s\rangle}^{2} V_{s, j} / \sum_{j^{\prime}=1}^{d^{\prime}} V_{s, j^{\prime}}$. The overall problem dimension is then $d=22$. We used the same marginal variances when running multiple independent replications.

Figure 1 presents two log-log plots of the RMSE for the estimators of $\xi$ using MC or RQMC, where we estimated the RMSEs from $10^{3}$ independent replications. Each estimator is based on a total of $n=r m$ evaluations of the response function $w_{Y}$. For RQMC, $m$ represents the point-set size, and $r$ is the number of randomizations. For each version of the model dimension $d(=3$ or 22), we compare two RQMC estimators of $\xi$, denoted RQMCv: $d$ for $\mathrm{v}=1$ or 2 in the figure. RQMC1: $d$ is the estimator $\bar{\xi}_{\mathrm{RQMC}, m, r}$ in (20), and RQMC2: $d$ is the estimator $\widetilde{\xi}_{\mathrm{RQMC}, m, r}$ in (28). In the following, we often simplify notation by omitting the ": $d$ " in the discussions. For RQMC, we used a lattice point set with a random shift modulo 1 for randomization, utilizing the code of [12]. We also ran experiments employing a Sobol' point set with a random digital shift ([28] shows this is a good practical choice), and the results (not shown) are qualitatively similar.

For $\mathrm{MC}$, we also computed two different estimators, denoted $\mathrm{MCv}$ for $\mathrm{v}=1$ or 2 in the figure. (For MC, we plot the results for only $d=22$ and not for $d=3$ because the results are stochastically equivalent.) The $\mathrm{v}=1$ estimator (i.e., MC1) averages $r$ independent $p$-quantile estimators, where each 

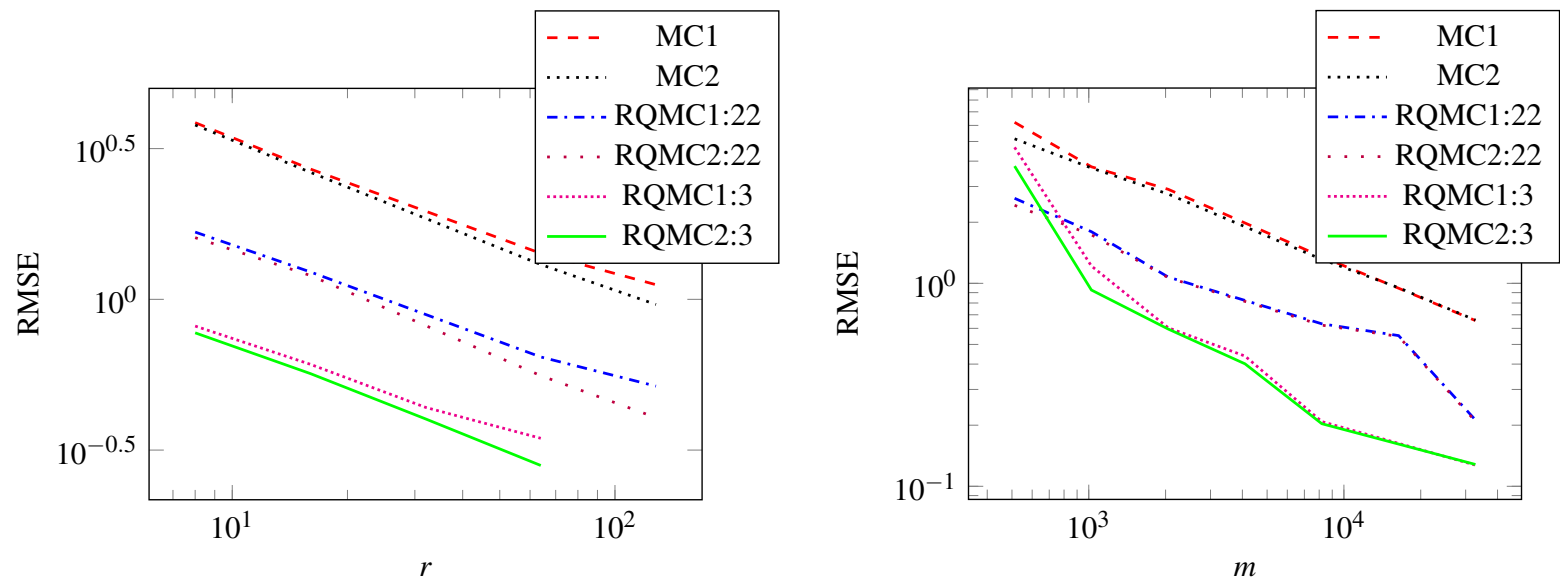

Figure 1: The left plot shows RMSE for fixed $m=4096$ as $r$ increases, and the right graph displays RMSE for fixed $r=32$ as $m$ increases. Both plots have log-log scale.

estimator is calculated by inverting a CDF estimator based on a sample of size $m$. The $\mathrm{v}=2$ estimator computes a single $p$-quantile estimator by inverting a CDF estimator from all $n=r m$ outputs.

The graph on the left side of Figure 1 has fixed $m=4096$ and $r$ increasing, and the plot on the right has fixed $r=32$ and $m$ increasing. (We chose $r=32$ for the right plot as this corresponds to the common (but sometimes inadequate) rule of thumb that the asymptotics of a CLT roughly start holding for sample sizes at least 30.) To interpret the left plot of Figure 1, recall that MSE decomposes as bias squared plus variance. For RQMC2, RMSE shrinks at rate $O\left(r^{-1 / 2}\right)$ as $r$ grows with $m$ fixed by (33). But for RQMC1, (24) shows that the bias contribution to MSE does not change as $r$ increases with $m$ fixed. (For $v=1,2$, the RMSE of MCv behaves like that of RQMCv.) For small $r$, the variance dominates the MSE for $\mathrm{v}=1$, and the RMSEs for $\mathrm{v}=1$ and 2 are close. For $\mathrm{v}=1$, as $r$ grows, the variance shrinks, but the bias does not change, so the bias eventually dominates the RMSE, and the RMSEs for $\mathrm{v}=1$ and 2 then separate for large $r$. For RQMC, the curves for $d=3$ and $d=22$ are qualitatively similar, but the RMSEs for $d=3$ are smaller, where the plots for $v=2$ have equal slope but different intercepts.

For the right plot of Figure 1, for MC or RQMC, the $\mathrm{v}=1$ and 2 estimators' RMSEs differ for small $m$. But as $m$ grows, we see that the RMSE for $\mathrm{v}=1$ and 2 eventually merge.

\section{CONCLUDING REMARKS}

We considered two different RQMC quantile estimators: $\bar{\xi}_{\mathrm{RQMC}, m, r}$ in (20) and $\widetilde{\xi}_{\mathrm{RQMC}, m, r}$ in (28). In both cases, we considered a low-discrepancy point set $\mathscr{P}_{m}$ of fixed size $m$ and let the number $r$ of randomizations grow large. Unfortunately, the first estimator $\bar{\xi}_{\mathrm{RQMC}, m, r}$ then converges to the wrong value as $r \rightarrow \infty$, as seen in (23). Thus, the CI $J_{\mathrm{RQMC}, m, r}$ in (26) may have poor coverage as $r \rightarrow \infty$.

In contrast, our other RQMC quantile estimator $\widetilde{\xi}_{\mathrm{RQMC}, m, r}$ in (28) does converge (in RMSE) to the desired value $\xi$. We are currently investigating methods to build an asymptotically valid CI for $\xi$ based on $\widetilde{\xi}_{\mathrm{RQMC}, m, r}$. 
To try to gain the faster convergence rate of QMC, we may want to also let $m$ grow large, which may then lead to the first estimator $\bar{\xi}_{\mathrm{RQMC}, m, r}$ in (20) converging to $\xi$. We are currently working on formulating asymptotic regimes in which both $m \rightarrow \infty$ and $r \rightarrow \infty$.

\section{ACKNOWLEDGMENTS}

This work has been supported in part by the National Science Foundation under Grant No. CMMI1537322. Any opinions, findings, and conclusions or recommendations expressed in this material are those of the authors and do not necessarily reflect the views of the National Science Foundation.

\section{References}

[1] A. Alban, H. Darji, A. Imamura, and M. K. Nakayama. Efficient Monte Carlo methods for estimating failure probabilities. Reliability Engineering and System Safety, 165:376-394, September 2017.

[2] A. N. Avramidis and J. R. Wilson. Correlation-induction techniques for estimating quantiles in simulation. Operations Research, 46:574-591, 1998.

[3] R. R. Bahadur. A note on quantiles in large samples. Annals of Mathematical Statistics, 37(3):577-580, 1966.

[4] P. Billingsley. Probability and Measure. John Wiley and Sons, New York, 3rd edition, 1995.

[5] D. A. Bloch and J. L. Gastwirth. On a simple estimate of the reciprocal of the density function. Annals of Mathematical Statistics, 39:1083-1085, 1968.

[6] F. Chu and M. K. Nakayama. Confidence intervals for quantiles when applying variance-reduction techniques. $A C M$ Transactions On Modeling and Computer Simulation, 22(2):10:1-10:25, 2012.

[7] R. Cranley and T. N. L. Patterson. Randomization of number theoretic methods for multiple integration. SIAM Journal on Numerical Analysis, 13(6):904-914, 1976.

[8] D. A. Dube, R. R. Sherry, J. R. Gabor, and S. M. Hess. Application of risk informed safety margin characterization to extended power uprate analysis. Reliability Engineering and System Safety, 129:19-28, 2014.

[9] Z. He and X. Wang. Convergence of randomized quasi-Monte Carlo sampling for value-at-risk and conditional value-atrisk. arXiv:1706.00540, 2017

[10] X. Jin and A. X. Zhang. Reclaiming quasi-Monte Carlo efficiency in portfolio value-at-risk simulation through Fourier transform. Management Science, 52(6):925-938, 2006.

[11] P. Jorion. Value at Risk: The New Benchmark for Managing Financial Risk. McGraw-Hill, New York, 3rd edition, 2007.

[12] F. Y. Kuo and D. Nuyens. Application of quasi-monte carlo methods to elliptic PDEs with random diffusion coefficients - a survey of analysis and implementation. Foundations of Computational Mathematics, 16(6):1631-1696, 2016.

[13] Y. Lai and K.S. Tan. Simulation of nonlinear portfolio value-at-risk by monte carlo and quasi-monte carlo methods. In M. Holder, editor, Financial Engineering and Applications. ACTA Academic Press, 2006.

[14] P. L'Ecuyer, D. Munger, and B. Tuffin. On the distribution of integration error by randomly-shifted lattice rules. Electronic Journal of Statistics, 4:950-993, 2010.

[15] C. Lemieux. Monte Carlo and Quasi-Monte Carlo Sampling. Series in Statistics. Springer, New York, 2009.

[16] C. Lemieux and P. L'Ecuyer. Selection criteria for lattice rules and other low-discrepancy point sets. Mathematics and Computers in Simulation, 55(1-3):139-148, 2001.

[17] W.-L. Loh. On the asymptotic distribution of scrambled net quadrature. Annals of Statistics, 31(4):1282-1324, 2003.

[18] M. K. Nakayama. Estimating a failure probability using a combination of variance-reduction tecniques. In L. Yilmaz, W. K. V. Chan, I. Moon, T. M. K. Roeder, C. Macal, and M. D. Rossetti, editors, Proceedings of the 2015 Winter Simulation Conference, pages 621-632, Piscataway, New Jersey, 2015. Institute of Electrical and Electronics Engineers. 
[19] H. Niederreiter. Random Number Generation and Quasi-Monte Carlo Methods, volume 63. SIAM, Philadelphia, 1992.

[20] A. B. Owen. Randomly permuted (t,m,s)-nets and (t,s)-sequences. In Monte Carlo and Quasi-Monte Carlo Methods in Scientific Computing: Lecture Notes in Statistics, volume 106, pages 299-317. Springer, 1995.

[21] A. B. Owen. Monte Carlo variance of scrambled net quadrature. SIAM Journal of Numerical Analysis, 34:1884-1910, 1997.

[22] A. B. Owen. Scrambled net variance for integrals of smooth functions. Annals of Statistics, 25(4):1541-1562, 1997.

[23] A. B. Owen. Scrambling Sobol' and Niedeerreiter-Xing points. Journal of Complexity, 14(4):466-489, 1998.

[24] A. Papageorgiou and S. H. Paskov. Deterministic simulation for risk management. Journal of Portfolio Management, 25(5):122-127, 1999.

[25] R. J. Serfling. Approximation Theorems of Mathematical Statistics. John Wiley and Sons, New York, 1980.

[26] R. R. Sherry, J. R. Gabor, and S. M. Hess. Pilot application of risk informed safety margin characterization to a total loss of feedwater event. Reliability Engineering and System Safety, 117:65-72, 2013.

[27] I. H. Sloan and S. Joe. Lattice Methods for Multiple Integration. Carendon Press, Oxford, UK, 1994.

[28] B. Tuffin. On the use of low discrepancy sequences in Monte Carlo methods. Monte Carlo Methods and Applications, 2(4):295-320, 1996.

[29] B. Tuffin. Variance reduction order using good lattice points in Monte Carlo methods. Computing, 61(4):371-378, 1998.

[30] B. Tuffin. Randomization of quasi-monte carlo methods for error estimation: Survey and normal approximation. Monte Carlo Methods and Applications, 10(3-4):617-628, 2004.

[31] U.S. Nuclear Regulatory Commission. Final safety evaluation for WCAP-16009-P, revision 0, "realistic large break LOCA evaluation methodology using automated statistical treatment of uncertainty method (ASTRUM)" (TAC no. MB9483). Technical report, U.S. Nuclear Regulatory Commission, Washington, DC, 2005. https://www.nrc.gov/docs/ML0509/ML050910159.pdf.

[32] U.S. Nuclear Regulatory Commission. Acceptance criteria for emergency core cooling systems for light-water nuclear power reactors. Title 10, Code of Federal Regulations §50.46, NRC, Washington, DC, 2010.

[33] U.S. Nuclear Regulatory Commission. Applying statistics. U.S. Nuclear Regulatory Commission Report NUREG-1475, Rev 1, U.S. Nuclear Regulatory Commission, Washington, DC, 2011. 